\title{
New Media
}

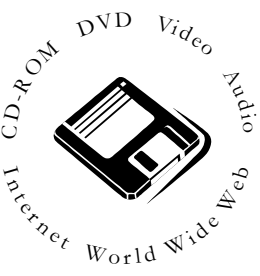

\section{[1] Web-Based Examinations Come of Age}

Certification programs are commonly embraced in the computer community, where one can obtain formal credentials from Microsoft, CompTIA or other authorities in a specific area of technical expertise such as knowing how to use Linux or Windows 2000, or how to program in C or HTML. Most certification program examinations cost between $\$ 100$ and \$200 US and are administered by computer at proctored testing sites around North America just like the current LMCC examination facing Canadian medical graduates.

Brainbench.com and its poorer cousin ecertifications.com are websites offering inexpensive but unproctored web-based examinations for individuals seeking certification in a particular knowledge domain. Brainbench is the best developed, offering some 350 examinations covering everything from Internet security to telephone etiquette (the later and similar office-related examinations being aimed at evaluating potential filing clerks, receptionists and secretaries). While the selection of examinations is richest in the computer arena, it also offers a surprising number of health-care related examinations aimed at paramedics and nurses who wish to demonstrate their continuing professional development. Some of the certification exams are particularly easy for anesthesiologists - I effortlessly passed examinations on medical terminology, CPR and a number of other medical topics, although my biochemistry knowledge was way too faded for me to pass that particular exam.

Both web-based certification programs are quite inexpensive - examinations are currently free at ecertifications.com, and cost \$20 US each at Brainbench.com, with an option for a $\$ 100$ US annual subscription allowing as many examinations as you wish to take. (Until recently examinations were also free at Brainbench.) In the case of the ecertifications site, successful candidates are offered an instant e-certificate in PDF format that is almost suitable for framing (provided you print it out on certificate-quality paper); suc- cessful Brainbench candidates are sent a real signed certificate in the mail in addition to having their certifications displayed online by entering the appropriate transcript number (such as 1791881 , my number).

Brainbench also has a comprehensive discussion forum whereby one can make suggestions or do a little bragging. A number of individuals, obviously technically well-rounded, have collected large numbers of Brainbench certificates in what has become a friendly contest among folks across the globe to collect the largest number of unique certifications. However, enthusiasm for collecting these certifications fell dramatically once Brainbench started charging. Regardless, so far a small handful of individuals among the over three million Brainbench users have broken the rarified " 100 certifications" barrier.

The Brainbench site also offers a number of useful educational resources for individuals who wish to prepare for an examination. These include information on newsgroups, web sites and discussion forums that may be of value. In addition, Brainbench offers sample examinations (based on retired questions) that may be purchased.

The unproctored character of the examination process with Brainbench and ecertification.com is a concern to some, as cheating is very easy. Also, the examinations are "open-book" in nature, and allow one to draw on paper and electronic resources as needed. This has led a number of people to question the value of certifications of this kind. However, employers can eliminate these concerns by simply testing candidates at their own premises under the watchful eye of a local proctor. Also, there is no reason why a proctoring process could not be added in the same way as a number of open universities operate, with candidates arranging for a suitable proctor (such as a high-school principal) to be present to attest that the examination was completed honestly. Indeed, with web cam technology and other remote monitoring technologies now on the horizon, there is little reason why the proctor will need to be physically close to the test candidate! 
I fully expect that this technology will soon be adapted for medical education purposes. For instance, clinical clerks might take the written portion of their anesthesia examination in this way, under the proctorship of the departmental secretary. If several universities were to coordinate their efforts, the size of the question pool could be made quite large and all questions could be scientifically validated - something rarely done in current anesthesia clerkship examinations. Universities could also see how their students do relative to other universities and take corrective actions where deficiencies are identified.

The possibilities offered by web technology in education is truly striking. For more insights into what one can expect in the future, take a look at some of the efforts also underway on the web at medschool.com, freetutorials.com, med2learn.com, click2learn.com, blackboard.com and worldwidelearn.com.

D. John Doyle MD PhD FRCPC

Toronto, Ontario

\section{[2] Software Review}

StarOffice Release 5.2

http://www.sun.com/software/star/staroffice/ StarOffice ${ }^{\mathrm{TM}} 5.2$ suite is a "free, full-featured, integrated, interoperable office suite" from Sun Microsystems offering "rich, easy-to-use office productivity tools in a unified desktop environment". StarOffice can be downloaded without charge for the Windows environment and other platforms (e.g., Linux). It takes up much less hard-disk space than Microsoft Office, and, being free, is certainly much cheaper.

According to Sun Microsystems, the office suite "provides complete functionality including word processing, spreadsheet, presentation graphics, drawing and photo editing, database, one-click HTML creation and editing, email, browser, internet newsgroup software, schedule and calendar, clip art, templates and more". Comparable in functionality to Microsoft Office, its user interface is intuitive and straightforward. Indeed, StarOffice is "interoperable" with Microsoft Office file formats such as ".doc", ".ppt", and "xls". StarOffice "includes an import/export feature that provides seamless interoperability with Microsoft Office" so that one can easily open and edit existing Microsoft Word, Excel, and PowerPoint files in StarOffice.

One can also save StarOffice documents, spreadsheets, and presentations as Microsoft Office files.

A companion site at http://www.staroffice.com offers a user support discussion group, a developer sup- port discussion group as well as information on the many books available to learn about StarOffice. Also available at this site are "portal services" such as free email addresses of the form you@staroffice.com, as well as free web-based storage space for your documents.

A useful list of frequently asked questions is available at http://www.sun.com/software/star/staroffice/5.2/faqs-general.html

For technical types, the source code for StarOffice software is available at OpenOffice.org. Sun has also made the StarOffice APIs and XML file formats available as well, "in an effort to drive standardization across office productivity suites". This means that developers around the world can even modify StarOffice "to best suit their needs, whether to improve their own products, build new value-added products on top of the StarOffice suite, improve existing technology in StarOffice software, or contribute new StarOffice components to the open source community". This move opens up the market to unlimited possibilities for innovation that simply are not possible with closed systems such as Microsoft Office.

I had no trouble downloading the $77 \mathrm{MB}$ installation file and setting up the suite. Still, the file size is daunting if you do not have a high-speed Internet connection, so some users might prefer to order the CD version from Sun. I found the program to be intuitive and easy to use but with some unexpected differences. For instance, unlike Microsoft Office, where users typically use Word or PowerPoint as standalone applications, StarOffice uses an integrated design. That is, from the StarOffice desktop, one creates different types of documents by selecting the document type desired from a pull-down menu.

While version 5.2 of StarOffice includes more than 200 fixes and enhancements over the previous release, there are still some restrictions and problems, particularly in effortlessly exchanging StarOffice documents with Microsoft Office. (For more details, read the review at: http: / /www.networkcomputing.com/unixworld/1118/1118uw.html).

Despite these limitations, StarOffice 5.2 is a solid piece of software at a price that is hard to argue with.

\section{John Doyle MD PhD FRCPC}

Toronto, Ontario

\section{Erratum}

In the article entitled: "Separation of omphalopagus conjoined twins using combined caudal epidural general anesthesia" published in the May 2001 issue (pages 478-82), the second author's name should have read: David D. Frankville. 